BMJ Open Sport \& Exercise Medicine

\title{
Exposure to physical and psychosocial stressors in relation to symptoms of common mental disorders among European professional football referees: a prospective cohort study
}

\author{
Özgür Kilic, ${ }^{1,2,3}$ Urban Johnson, ${ }^{4}$ Gino M M J Kerkhoffs, ${ }^{1,2,3}$ Philippe Rosier, ${ }^{5,6}$ \\ Vincent Gouttebarge $\mathrm{e}^{1,2,3,7}$
}

\begin{abstract}
To cite: Kilic 0̈, Johnson U, Kerkhoffs GMMJ, et al. Exposure to physical and psychosocial stressors in relation to symptoms of common mental disorders among European professional football referees: a prospective cohort study. BMJ Open Sport \& Exercise Medicine 2018;4:e000306. doi:10.1136/ bmjsem-2017-000306
\end{abstract}

Accepted 11 February 2018

Check for updates

${ }^{1}$ Department of Orthopaedic Surgery, Academic Medical Center, University of Amsterdam, Amsterdam Movement Sciences, Amsterdam, The Netherlands

${ }^{2}$ Academic Center for Evidence Based Sports Medicine (ACES), Academic Medical Center, Amsterdam, The Netherlands ${ }^{3}$ Amsterdam Collaboration on Health \& Safety in Sports (ACHSS), AMC/VUmc IOC

Research Center, Amsterdam,

The Netherlands

${ }^{4}$ Halmstad University, Halmstad, Sweden

${ }^{5}$ Royal Belgian Football Association, Brussels, Belgium ${ }^{6}$ Centre de Recherche Appliquée en Psychopédagogie de la Perception, University Fernando Pessoa, Porto, Portugal ${ }^{7}$ Division of Exercise Science and Sports Medicine, University of Cape Town, Cape Town, South Africa

Correspondence to Dr Vincent Gouttebarge; v.gouttebarge@amc.uva.nl

\section{ABSTRACT}

Objectives The study aim was to explore the association of physical and psychosocial stressors (severe injuries, surgeries, recent life events, social support) with one-season onset of symptoms of common mental disorders (CMDs) among European professional football referees.

Methods An observational prospective cohort study over a follow-up period of one season (2015-2016) was conducted among professional football referees from Belgium, Finland, France, Germany, Norway, Russia, Scotland and Sweden. Based on physical and psychosocial stressors as well as symptoms of CMD, an electronic questionnaire in English and French was set up and distributed by eight football federations involved.

Results The prevalence of symptoms of CMD ranged from $5.9 \%$ for distress to $19.2 \%$ for eating disorders. A higher number of severe injuries and a lower degree of satisfaction about social support were significantly related to the occurrence of symptoms of CMD with an OR of 2.63 and an $\mathrm{OR}$ of 1.10 , respectively.

Conclusion A higher number of severe injuries and a lower degree on satisfaction about social support were found to be significantly associated with the onset of symptoms of CMD among European professional football referees. Referees suffering from severe injuries were nearly three times more likely to report symptoms of anxiety and depression. Referees who reported a low satisfaction of social support were significantly more likely to report symptoms of eating disorder.

\section{INTRODUCTION}

Symptoms of common mental disorders (CMDs)—such as symptoms of distress, anxiety, depression or substance abuse/ dependence-are often comorbid and more frequently reported in young adult populations than at any other stage of the lifespan. ${ }^{1-4}$ Estimation of the WHO showed that the 12-month prevalence of any symptoms of CMD ranged from $9 \%$ to $19 \%$ across

\section{What are the new findings?}

- This study presents the results of the first prospective study that has explored the longitudinal interaction of physical and psychosocial stressors with menta health problems among professional referees.

- The prevalence of symptoms of common mental disorders (CMDs) ranged from $5.9 \%$ for distress to $19.2 \%$ for eating disorders. Nearly half of the participants reported symptoms of CMD at baseline.

- Two stressors showed a significant relationship with the occurrence of symptoms of CMD, namely severe injuries and a lower degree of satisfaction about social support with an OR of 2.63 and 1.10 respectively.

How might it impact on clinical practice in the near future?

- Thestudy emphasises the need for an interdisciplinary medical approach among professional football referees, focusing on the physical and psychological aspects.

- These results create the opportunity for the clinical practice to identify referees at risk of developing symptoms of CMD timely.

- Such an interdisciplinary medical approach could prevent symptoms of CMD from getting worse and consequently improve the performance of referees.

European countries, while a recent systematic meta-analysis concluded that nearly one out of five adults experienced a CMD within the past 12 months and $29 \%$ across their lifetime. ${ }^{56}$

The awareness about symptoms of CMD within professional football (soccer) has been risen, prevalence rates reaching up to $43 \%$ for symptoms of anxiety/depression among European professional footballers. ${ }^{7}$ Among current professional footballers, several 
physical and psychosocial stressors were found related to the occurrence of symptoms of CMD, especially severe musculoskeletal injuries, surgeries and adverse life events. ${ }^{7-10}$ An earlier study among professional European football players showed that players with severe musculoskeletal injuries were nearly seven times more likely to report symptoms of CMDs than players with no injury. ${ }^{11}$ Among central and assistant referees, another essential actor within professional football, the 4-week prevalence of symptoms of CMD (self-reported and not clinically diagnosed) ranged from $6 \%$ for distress to $31 \%$ for eating disorders, and the one-season incidence from $8 \%$ for adverse alcohol use to $29 \%$ for eating disorders. ${ }^{12}$

In contrast to the available scientific evidence among current professional football players, scientific evidence about the stressors that play a role in the onset of symptoms of CMD among professional football referees, is not available yet. Based on a similar study among players, a plausible assumption is that physical and psychosocial stressors such as severe injuries, surgeries, adverse life events and lower social support are likely to induce symptoms of CMD among professional football referees. Accordingly, the aim of the present study was to explore the association of physical and psychosocial stressors (severe injuries, surgeries, recent life events, social support) with the one-season onset of symptoms of CMD (distress, anxiety/depression, sleeping disturbance, eating disorders, adverse alcohol use) among European professional football referees.

\section{METHODS \\ Design}

An observational prospective cohort study with three measurements over a follow-up period of one season (from July 2015 to May 2016) was conducted among professional football referees. The present research was conducted in accordance with the Declaration of Helsinki. $^{13}$

\section{Study setting and participants}

Because of their interest in the mental health of their referees, the football federations from Belgium, Finland, France, Germany, Norway, Russia, Scotland and Sweden participated in the study and assisted in the recruitment of participants. Inclusion criteria were: (1) being active as central or assistant referee in a professional football league; (2) being 18 years old or older and (3) being able to read and comprehend texts fluently in either English or French. Sample size calculation indicated that at least 138 participants were needed to detect with a precision of $5 \%$ that 1 out of 10 professional football referees suffer from a mental health condition (power of $80 \%$; $95 \%$ CI) ${ }^{14}$ Expecting a response rate of around $40 \%$ (based on recent similar studies in professional sports) and a lost to follow-up at $20 \%$, we intended to invite at least 440 referees. The eight European football federations were asked to invite between 40 and 80 referees per country fulfilling the inclusion criteria. Contact details of potential participants were blinded to the responsible researchers for reasons of privacy and confidentiality. Potential participants were invited by the football federations in June-July 2015.

\section{Physical and psychosocial stressors (independent variables)}

Severe injuries: The total number of severe injuries in the previous 6 months was assessed through a single question (eg, 'How many severe injuries have you had in the previous 6 months'). In our study, severe musculoskeletal injury was defined as an injury that involved the musculoskeletal system (bone, joint, ligament, muscles, tendons, etc) and occurred during refereeing and led to either training or match absence for more than 28 days (definition being clearly stated to the participants). ${ }^{15}$

- Surgeries: The total number of surgeries in the previous 6 months was examined through a single question (eg, 'How many surgeries have you had in the previous 6 months?').

- Recent life events: Based on the validated Social Athletic Readjustment Rating Scale, the occurrence of life events (eg, 'Death of spouse', 'Change in financial state') in the previous 6 months was explored by 13 single questions (yes or no). ${ }^{16}$ A total score was calculated by summing up the life events occurred.

- Social support: The validated Social Support Questionnaire (short form) was used to measure social support (parents, other family members, friends, colleagues, etc) in the previous 6 months. ${ }^{17}$ Based on six items, a total score is calculated, with a higher score representing a lower degree of satisfaction with social support. ${ }^{17}$

\section{Symptoms of CMDs (dependent variables)}

- Distress: Distress in the previous 4 weeks (baseline measurement) and in the previous 6 months (followup measurements) was measured using the Distress Screener (three items scored on a 3-point scale) which is based on the four-dimensional symptom questionnaire (4DSQ) (eg, 'Did you recently suffer from worry?'). ${ }^{18}$ The $4 \mathrm{DSQ}$, that is, Distress Screener has been validated in several languages, including English and French (test-retest coefficients $\geq 0.89$; criterion-related validity: area under receiver operating characteristic (ROC) curve $\geq 0.79$ ). ${ }^{18} 19$ A total score ranging from 0 to 6 was obtained by summing up the answers on the three items, a score of 4 or more indicating the presence of distress.

- Anxiety/depression: The 12-Item General Health Questionnaire (GHQ-12) was used to assess symptoms of anxiety/depression in the previous 4 weeks (baseline measurement) and in the previous 6 months (follow-up measurements) (eg, 'Have you recently felt under strain?').$^{20}$ The GHQ-12 has been validated in several languages, including English and French (criterion-related validity: sensitivity $\geq 0.70$, 
specificity $\geq 0.75$, area under ROC curve $\geq 0.83$ ). ${ }^{20}$ Based on the traditional scoring system (4-point scale with two favourable and two unfavourable answers), a total score ranging from 0 to 12 was calculated by summing up the answers on the 12 items, with a score of 2 or more indicating signs of anxiety/depression (area under curve $=0.88$ ) ${ }^{20}$

- Sleeping disturbance: Based on the PatientReported Outcomes Measurement Information System (PROMIS; short form) sleep disturbance in the previous 4 weeks (baseline measurement) and in the previous 6 months (follow-up measurements) was assessed through four single questions (eg, 'Did you recently have some problem to sleep?') scored on a 4-point scale (0 for favourable answers, 1 for unfavourable answers). ${ }^{21}$ The PROMIS has been validated in several languages, including English and French (construct validity: product-moment correlations 20.96 ) (for detailed information, see www.nihpromis.org). A total score ranging from 1 to 20 is obtained by summing up the answers to the four questions, a score of 12 or less indicating the absence of sleep disturbance and a score of 13 or more indicating the presence of sleep disturbance. ${ }^{21}$

- Eating disorders: The Eating disorder Screen for Primary care (5-item questionnaire scored as 'yes' or 'no'; ' 0 ' for favourable answer and ' 1 ' for unfavourable answer) was used as a screening instrument to detect eating disorders in the previous 4 weeks (baseline measurement) and in the previous 6 months (follow-up measurements) (eg, 'In the past 4 weeks, were you satisfied with your eating patterns?'). ${ }^{22}$ The Eating disorder Screen for Primary care has been validated in several languages, including English and French (criterion-related validity: sensitivity $100 \%$, specificity 0.71$).{ }^{22}$ A total score ranging from 0 to 5 is obtained by summing up the answers on the five items, a score of 2 or more indicating the presence of eating disorders. ${ }^{22}$

- Adverse alcohol use: Level of alcohol consumption at present time (baseline measurement) and in the previous 6 months (follow-up measurements) was detected using the 3-Item Alcohol Use Disorders Identification Test (AUDIT-C) (eg, 'How many standard drinks containing alcohol do you have on a typical day?'). ${ }^{23}$ The AUDIT-C has been validated in several languages, including Danish (criterionrelated validity: area under ROC curve 0.700.97). ${ }^{23} 24$ A total score ranging from 0 to 12 was obtained by summing up the answers on the three items (five answer categories per item), a score of 5 or more indicating the presence of adverse alcohol behaviour.

\section{Procedures}

A baseline and two follow-up electronic questionnaires (similar to each other) available in English and French were set up (FluidSurveys), involving the following descriptive variables: age, height, body mass, duration of professional football referee career, main function (central or assistant referee), level of education and any occupation beside refereeing (hours per week). Each questionnaire took about 15-20 min to complete. Information about the study was sent per email to potential participants by the European football federations. Interested participants gave their informed consent and were given access to the baseline online questionnaire. At the end of the baseline questionnaire, participants could leave their email address and give their informed consent for the follow-up online questionnaires, which were sent per email 5 and 10 months later. Participants were asked to complete the questionnaires within 1 week. Reminders at baseline and follow-up were sent after 1 and 2 weeks. The responses to baseline and follow-up questionnaires were coded and anonymised for reasons of privacy and confidentiality. The completed electronic questionnaires were saved automatically on a secured electronic server accessible only by the principal researcher. Referees participated voluntarily and did not receive any reward for their participation.

\section{Statistical methods}

All data analyses were performed using the statistical software IBM SPSS Statistics V.23.0 for Windows. Descriptive data analyses (mean, SD, frequency, range) were performed for the different variables involved in our study. Prevalence (expressed as a percentage) was calculated as the proportion of the number of participants with a given symptom of CMD relative to the total number of participants. ${ }^{14}$ Univariate and multivariate (all stressors combined) logistic regression analyses, expressed as OR and related $95 \% \mathrm{CI}$, were performed to explain the potential relationship between a stressor (severe injuries, surgeries and life events as continuous variable) and the presence/absence of the outcome measures under study. ${ }^{14}$

\section{RESULTS \\ Participants}

The football federations from Belgium, Finland, France, Germany, Norway, Russia, Scotland and Sweden contacted a sample of 646 professional football referees. Of those, 391 gave their written informed consent to participate in the study (response rate of $61 \%$ ), while 292 completed the follow-up questionnaires (follow-up rate of $75 \%$ ). No statistically significant difference was found between non-responders and responders at follow-up. The mean age of the 391 participants at baseline was 33 years old $(\mathrm{SD}=7)$ and they were refereeing in professional football for 7 years on average ( $45 \%$ as central referee; $55 \%$ as referee assistant). More than 9 out of 10 participants were employed aside refereeing, working on average for 36 hours a week in addition to refereeing. Baseline prevalence of symptoms of CMD ranged from nearly $6 \%$ for 


\begin{tabular}{|c|c|c|c|}
\hline Characteristics & $\begin{array}{l}\text { Total } \\
(\mathrm{N}=391)\end{array}$ & $\begin{array}{l}\text { No CMD } \\
\text { (N=215) }\end{array}$ & $\begin{array}{l}\text { CMD } \\
(\mathrm{N}=175)\end{array}$ \\
\hline Age (in years; mean $\pm S D$ ) & $33 \pm 7$ & $33 \pm 6$ & $34 \pm 7$ \\
\hline Height (in cm; mean $\pm S D$ ) & $180 \pm 7$ & $181 \pm 7$ & $180 \pm 7$ \\
\hline Weight (in kg; mean $\pm S D$ ) & $76 \pm 9$ & $75 \pm 9$ & $76 \pm 8$ \\
\hline $\begin{array}{l}\text { Duration refereeing career (in years; } \\
\text { mean } \pm S D \text { ) }\end{array}$ & $7 \pm 5$ & $7 \pm 5$ & $7 \pm 6$ \\
\hline \multicolumn{4}{|l|}{ Main refereeing function (\%) } \\
\hline Central referee & 45 & 46 & 44 \\
\hline Assistant referee & 55 & 53 & 56 \\
\hline \multicolumn{4}{|l|}{ Educational level (\%) } \\
\hline Nursery/elementary school & 2 & 3 & 1 \\
\hline High school & 15 & 12 & 18 \\
\hline Vocational/technical school & 9 & 8 & 12 \\
\hline College, university (equivalent) & 74 & 77 & 70 \\
\hline Employed aside refereeing (\%) & 92 & 95 & 90 \\
\hline Working hours per week (mean \pm SD) & $36 \pm 8$ & $37 \pm 8$ & $36 \pm 8$ \\
\hline \multicolumn{4}{|l|}{ Symptoms of CMD \% } \\
\hline Distress & 5.9 & & \\
\hline Anxiety/depression & 11.8 & & \\
\hline Sleeping disturbance & 9.1 & & \\
\hline Eating disorders & 19.2 & & \\
\hline Adverse alcohol use & 16.5 & & \\
\hline \multicolumn{4}{|c|}{ Comorbidity of simultaneous symptoms of CMD \% } \\
\hline Two symptoms & 7.7 & & \\
\hline Three symptoms & 2.0 & & \\
\hline Four symptoms & 1.0 & & \\
\hline \multicolumn{4}{|l|}{ Severe injuries (\%) } \\
\hline None & 82 & 82 & 82 \\
\hline 1 or 2 & 18 & 17 & 17 \\
\hline Three or more & 1 & 1 & 1 \\
\hline \multicolumn{4}{|l|}{ Surgeries (\%) } \\
\hline None & 96 & 97 & 96 \\
\hline 1 or 2 & 4 & 3 & 4 \\
\hline Three or more & 0 & 0 & 1 \\
\hline \multicolumn{4}{|l|}{ Recent life events (\%) } \\
\hline None & 50 & 49 & 45 \\
\hline 1 or 2 & 42 & 42 & 47 \\
\hline Three or more & 8 & 9 & 8 \\
\hline Social support score (mean $\pm S D)$ & $11 \pm 4$ & $10 \pm 4$ & $12 \pm 5$ \\
\hline
\end{tabular}

CMD, common mental disorder.

distress to $19 \%$ for eating disorders. All characteristics of the participants are presented in table 1 .

\section{Association of stressors with symptoms of CMDs}

The prevalence of symptoms of CMD ranged from 5.9\% for distress to $19.2 \%$ for eating disorders. Among professional football referees, a higher number of severe injuries and a lower degree on satisfaction about social support were related to the occurrence of symptoms of CMD. The multivariate analysis (with $95 \% \mathrm{CI}$ ) for the relationship between severe injuries and symptoms of anxiety/ depression presented an OR of 2.63 (1.14 to 6.10). An univariate and multivariate analysis between satisfaction of social support and symptoms of eating disorders resulted, respectively, in an OR of 1.10 (1.01 to 1.21) and an OR of 1.10 (1.00 to 1.21). These statistics show that professional football referees exposed to severe injuries and/or exhibit a lower satisfaction of social support are more likely to report symptoms of CMD (especially symptoms of anxiety/depression and eating disorders) by comparison with referees who are less exposed or unexposed. All relationships between stressors and symptoms of CMD are represented in table 2.

\section{DISCUSSION}

The prevalence of symptoms of CMD ranged from 5.9\% for distress to $19.2 \%$ for eating disorders. Two stressors were found to be significantly associated with the onset of symptoms of CMD. These stressors were severe injuries and a low satisfaction of social support with an OR of 2.63 (multivariate analysis) and 1.10 (univariate and multivariate analyses), respectively. Professional football referees who reported a low satisfactions of social support or severe injuries were almost 0.10 to 3 times more likely to report symptoms of CMD.

\section{Methodological considerations}

A potential limitation of this study worth mentioning is that the measurement of symptoms of CMD and related stressors was done through self-report. One might logically assume that symptoms of CMD assessed by medical professionals, concerning a rather taboo matter like symptoms of CMD, might have led to less subjective information. As symptoms of CMD is a rather taboo subject, one might assume that the prevalence of symptoms of CMD within this study might be underestimated. However, the present study was carried out with anonymous recruitment procedures and electronic survey combined with validated scales, which may have limited the influence of social desirability/undesirability. The above-mentioned assumption of underestimation cannot be sustained as there was no measure of social desirability/undesirability involved in our study. Another potential limitation is that the recruitment procedures were blinded to the research team for privacy and confidentiality reason. Consequently, a non-response analysis could not be conducted. Furthermore, participants (as in any scientific study) were free to be included in the study and thus self-selected. This might have led to selection bias as participants with more interest with symptoms of CMD might have been more likely to participate.

An important strength of this study is the prospective design as a longitudinal design allows the establishment of a causal exploration between symptoms of CMD and related stressors. 
Table 2 Relation (OR and 95\% Cl) between stressors and symptoms of common mental disorders among European professional football referees

\begin{tabular}{|c|c|c|c|c|c|}
\hline & Distress & Anxiety/depression & Sleeping disturbance & Eating disorders & Alcohol use \\
\hline \multicolumn{6}{|l|}{ Severe injuries } \\
\hline Univariate & 1.44 (0.66 to 3.16$)$ & $1.64(0.84$ to 3.17$)$ & 1.45 (0.72 to 2.92$)$ & 1.46 (0.79 to 2.69$)$ & 1.38 (0.59 to 3.24$)$ \\
\hline Multivariate & 2.07 (0.80 to 5.39$)$ & $2.63(1.14 \text { to } 6.10)^{*}$ & 2.04 (0.86 to 4.83$)$ & 1.04 (0.14 to 2.90$)$ & 2.03 (0.71 to 5.82$)$ \\
\hline \multicolumn{6}{|l|}{ Surgeries } \\
\hline Univariate & - & - & - & 1.85 (0.36 to 9.52$)$ & - \\
\hline Multivariate & - & - & - & $1.04(0.14$ to 8.00$)$ & - \\
\hline \multicolumn{6}{|l|}{ Life events } \\
\hline Univariate & $0.68(0.34$ to 1.36$)$ & 0.82 (0.52 to 1.30$)$ & 1.01 (0.68 to 1.50$)$ & 1.06 (0.79 to 1.43$)$ & 0.95 (0.56 to 1.63$)$ \\
\hline Multivariate & 0.65 (0.33 to 1.30$)$ & 0.77 (0.48 to 1.22$)$ & 0.96 (0.64 to 1.43$)$ & 1.02 (0.75 to 1.39$)$ & $0.92(0.54$ to 1.57$)$ \\
\hline \multicolumn{6}{|l|}{ Social support } \\
\hline Univariate & 1.04 (0.92 to 1.19$)$ & 1.04 (0.94 to 1.16$)$ & 1.05 (0.94 to 1.17$)$ & $1.10(1.01 \text { to } 1.21)^{\star}$ & $0.96(0.81$ to 1.13$)$ \\
\hline Multivariate & 1.05 (0.91 to 1.20$)$ & 1.04 (0.93 to 1.17$)$ & 1.05 (0.94 to 1.18$)$ & $1.10(1.00 \text { to } 1.21)^{\star}$ & 0.95 (0.79 to 1.13$)$ \\
\hline
\end{tabular}

\section{Implications}

As can be seen in table 2, a majority of the OR was near or $>1.0$; however, these were not found to be significant, which could be caused due to a power problem. This implicates that there might be possible association between the related stressors mentioned in this study and symptoms of CMD. However, one might also argue that referees have a high self-efficacy and are better resistant against psychological stressors and show less rapidly symptoms of CMD as referees have to be able to perform and withstand massive amounts of pressure during matches by athletes, coaches, fans and media, while a decision of a referee can have great consequences from economical and social perspective for clubs and fans as well as for athletes and teams. ${ }^{25}$ However, the predisposition of a low satisfaction of social support and severe injuries for symptoms of CMD among professional referees found in our study creates the opportunity to identify referees who are at risk of developing symptoms of CMD timely in order to prevent them from getting symptoms of CMD or prevent the worsening of these symptoms. Therefore, scientific information and further major studies on stressors causing symptoms of CMD are highly relevant.

Nearly half of the participants $(n=175)$ reported symptoms of CMD at baseline, which is worrying and should be taken into account as this can potentially influence the performance of referees. At baseline, prevalence of eating disorders reached around 20\%. This was contradictory to our beliefs that professional football referees, as other elite athletes, would be well aware that adverse alcohol use is not favourable to sport performances. Just like athletes, referees need psychological skills such as the ability to focus their attention and concentration, stay cool under pressure, cope with mistakes and adverse situations, and set realistic goals in order to perform successfully. ${ }^{25}$ If a referee does not feel ready physically and/or psychologically, it may lead to anxiety. ${ }^{25}$ As seen in our results, severe injuries increase the odds on symptoms of anxiety by 2.63 . Another factor that may play a role in referee efficacy is the perceived level of support/ non-support from significant others, which is similar to the satisfaction of social support mentioned in our study. ${ }^{25}$ In our results, low satisfaction of social support was associated with the onset of symptoms of CMD. As psychological factors are important for the performance of the referee as well as for the referees' self-efficacy, it is important to scientifically explore the relation between related stressors and symptoms of CMD by major studies.

An earlier study have shown that psychological factors such as low self-esteem, state anxiety, live event stress and daily hassle, worry and denial are linked to injury outcome. ${ }^{26}$ Many of these factors can be addressed through systematic interventions in order to reduce or minimise injury occurrence. ${ }^{26}$ Additionally, a previous study among professional European football players have shown that severe musculoskeletal injuries are likely to induce symptoms of common mental disorders. ${ }^{11}$ Another cross-sectional study among Danish professional football and handball players showed that injuries and adverse life events are associated with the occurrence of symptoms of common mental disorder. ${ }^{27}$ Our study showed that, next to low satisfaction of social support, injuries are also linked to the outcome of symptoms of CMD. This does not only emphasises the need for an interdisciplinary medical approach that focuses on the physical aspect as well as on the psychological aspect, but also creates the opportunity to identify referees who are at risk for developing symptoms of CMD in order to treat them timely.

\section{CONCLUSION}

The prevalence of self-reported symptoms of CMD ranged from $5.9 \%$ for distress to $19.2 \%$ for eating disorders. Two stressors were found to be significantly associated with the 
onset of symptoms of CMD, namely a higher number of severe injuries and a lower degree on satisfaction about social support. Referees suffering from severe injuries were nearly three times more likely to report symptoms of anxiety and depression. Referees who reported a low satisfaction of social support were significantly more likely to report symptoms of eating disorder. Adequate interventions and data concerning this matter is lacking and should be a focus of further scientific research in order to improve the awareness, performance and quality of life of professional football referees.

Acknowledgements The authors thank the Royal Belgian Football Association (Paul Allaerts), the Football Association of Finland (Johan Holmqvist), the French Football Federation (Pascal Garibian, Pierrick Guillemot), the German Football Association (Lutz Fröhlich, Hellmut Krug), the Football Association of Norway (Terje Hauge, Emil Waters), the Russian Football Union (Leonid Kaloshin, Sergey Tikhonov), the Scottish Football Association (John Fleming, Drew Herbertson, Steven McLean) and the Swedish Football Association (Anna Nyström). The authors are also very grateful to all referees for their participation in the study.

Contributors VG and GMMJK were responsible for the conceptualisation of the study's idea and data collection. ÖK was responsible for data analysis, interpretation of data and preparation of the manuscript. GMMJK, UJ, PR and VG were responsible for the critical review of the manuscript. All authors read and approved the final manuscript.

Funding The authors have not declared a specific grant for this research from any funding agency in the public, commercial or not-for-profit sectors.

Competing interests None declared.

Patient consent Obtained.

Ethics approval Ethical approval for this study was provided by the Medical Ethics Review Committee of the Academic Medical Center W15_050\#15.0061, Amsterdam, The Netherlands)

Provenance and peer review Not commissioned; externally peer reviewed.

Open Access This is an Open Access article distributed in accordance with the Creative Commons Attribution Non Commercial (CC BY-NC 4.0) license, which permits others to distribute, remix, adapt, build upon this work non-commercially, and license their derivative works on different terms, provided the original work is properly cited and the use is non-commercial. See: http://creativecommons.org/licenses/by-nc/4.0/

(C) Article author(s) (or their employer(s) unless otherwise stated in the text of the article) 2018. All rights reserved. No commercial use is permitted unless otherwise expressly granted.

\section{REFERENCES}

1. Association AP. Diagnostic and statistical manual of mental disorders. 5th edn. Washington: American Psychiatric Press, 2000.

2. King M, Nazareth I, Levy G, et al. Prevalence of common mental disorders in general practice attendees across Europe. $\mathrm{Br} J$ Psychiatry 2008;192:362-7.

3. Korten A, Henderson S. The Australian National Survey of Mental Health and Well-Being. Common psychological symptoms and disablement. Br J Psychiatry 2000;177:325-30.

4. Krueger RF, Caspi A, Moffitt TE, et al. The structure and stability of common mental disorders (DSM-III-R): a longitudinalepidemiological study. J Abnorm Psychol 1998;107:216-27.

5. Kessler RC, Aguilar-Gaxiola S, Alonso J, et al. The global burden of mental disorders: an update from the WHO World Mental Health (WMH) surveys. Epidemiol Psichiatr Soc 2009;18:23-33.
6. Steel Z, Marnane C, Iranpour C, et al. The global prevalence of common mental disorders: a systematic review and meta-analysis 1980-2013. Int J Epidemiol 2014;43:476-93.

7. Gouttebarge V, Backx FJ, Aoki H, et al. Symptoms of common mental disorders in professional football (Soccer) across five European countries. J Sports Sci Med 2015;14:811-8.

8. Gouttebarge V, Aoki H, Ekstrand J, et al. Are severe musculoskeletal injuries associated with symptoms of common mental disorders among male European professional footballers? Knee Surg Sports Traumatol Arthrosc 2016;24:3934-42.

9. Gouttebarge V, Aoki H, Kerkhoffs G. Symptoms of common mental disorders and adverse health behaviours in male professional Soccer players. J Hum Kinet 2015;49:277-86.

10. Ivarsson $\mathrm{A}$, Johnson $\mathrm{U}$, Lindwall $\mathrm{M}$, et al. Psychosocial stress as a predictor of injury in elite junior soccer: a latent growth curve analysis. J Sci Med Sport 2014;17:366-70.

11. Kiliç Ö, Aoki H, Goedhart E, et al. Severe musculoskeletal time-loss injuries and symptoms of common mental disorders in professional soccer: a longitudinal analysis of 12-month follow-up data. Knee Surg Sports Traumatol Arthrosc 2017.

12. Gouttebarge V, Johnson U, Rochcongar P, et al. Symptoms of common mental disorders among professional football referees: a one-season prospective study across Europe. Phys Sportsmed 2017:45:11-16

13. World Medical A. World Medical Association. World Medical Association Declaration of Helsinki: ethical principles for medical research involving human subjects. JAMA 2013;310:2191-4.

14. Woodward M. Epidemiology: study design and data analysis. 3rd edn. Boca Raton: CRC Press, Taylor \& Francis Group, 2014

15. Fuller CW, Ekstrand J, Junge $A$, et al. Consensus statement on injury definitions and data collection procedures in studies of football (soccer) injuries. Br J Sports Med 2006;40:193-201.

16. Bramwell ST, Masuda M, Wagner NN, et al. Psychosocial factors in athletic injuries: development and application of the social and athletic readjustment rating scale (SARRS). J Human Stress 1975;1:6-20.

17. Sarason IG, Sarason BR, Shearin EN, et al. A brief measure of social support: practical and theoretical implications. J Soc Pers Relat 1987;4:497-510.

18. Braam C, van Oostrom SH, Terluin B, et al. Validation study of a distress screener. J Occup Rehabil 2009;19:231-7.

19. Terluin B, van Marwijk HW, Adèr HJ, et al. The Four-Dimensional Symptom Questionnaire (4DSQ): a validation study of a multidimensional self-report questionnaire to assess distress, depression, anxiety and somatization. BMC Psychiatry 2006;6:34

20. Goldberg DP, Gater R, Sartorius N, et al. The validity of two versions of the GHQ in the WHO study of mental illness in general health care. Psychol Med 1997;27:191-7.

21. Yu L, Buysse DJ, Germain A, et al. Development of short forms from the PROMIS ${ }^{\mathrm{TM}}$ sleep disturbance and Sleep-Related Impairment item banks. Behav Sleep Med 2011;10:6-24.

22. Cotton MA, Ball C, Robinson P. Four simple questions can help screen for eating disorders. J Gen Intern Med 2003;18:53-6.

23. Dawson DA, Grant BF, Stinson FS, et al. Effectiveness of the derived Alcohol Use Disorders Identification Test (AUDIT-C) in screening for alcohol use disorders and risk drinking in the US general population. Alcohol Clin Exp Res 2005;29:844-54.

24. Cd M-G, Zuardi AW, Loureiro SR, et al. Alcohol Use Disorders Identification Test (AUDIT): an updated systematic review of psychometric properties. Psychology \& Neuroscience 2009;2:83-97.

25. Guillén F, Feltz DL. A conceptual model of referee efficacy. Front Psychol 2011;2:25.

26. Ivarsson A. Psychology of sport injury: prediction, prevention and rehabilitation in Swedish team sport athletes: Linnaeus University Dissertation No 219/2015

27. Kilic Ö, Aoki H, Haagensen R, et al. Symptoms of common mental disorders and related stressors in Danish professional football and handball. Eur J Sport Sci 2017;17:1328-34. 